\title{
Modeling of Stardust Entry at High Altitude, Part 1: Flowfield Analysis
}

\author{
Iain D. Boyd* \\ University of Michigan, Ann Arbor, Michigan 48109-2140 \\ and \\ Kerry A. Trumble \pm and Michael J. Wright \\ NASA Ames Research Center, Moffett Field, California 94035-1000
}

\section{DOI: $\underline{10.2514 / 1.37360}$}

\begin{abstract}
The Stardust sample return capsule entered the Earth's atmosphere at a very energetic velocity of $12.6 \mathrm{~km} / \mathrm{s}$. In the present study, both continuum (computational fluid dynamics) and particle (direct simulation Monte Carlo) methods are used to analyze the forebody flow of the Stardust sample return capsule at altitudes of 81 and $71 \mathrm{~km}$, where the flow is in the near-continuum regime. At the higher altitude, direct comparisons between baseline computational fluid dynamics and direct simulation Monte Carlo models give enormous differences in basic flowfield properties. To study the discrepancy between the solutions, a modified approach for determining the temperature used by computational fluid dynamics to control the dissociation and ionization reactions is investigated. The modified computational fluid dynamics and direct simulation Monte Carlo results are in significantly better agreement with each other, illustrating the strong sensitivity to chemistry modeling under these highly energetic conditions. Significant differences persist in temperatures near the capsule surface and in surface heat flux. Evaluation of local Knudsen numbers indicates that the flow experiences noncontinuum behavior in the shock front and at the capsule surface that explains the smaller heat flux predicted by direct simulation Monte Carlo. At the lower altitude, the flowfield results become less sensitive to details of the chemistry modeling, although noncontinuum effects are again predicted at the stagnation point.
\end{abstract}

\section{Introduction}

T HE Stardust payload was launched in February 1999 on a mission to use aerogel to collect samples of material from comet Wild-2. In January 2006, almost exactly seven years later, after collecting the cometary dust and returning to Earth, the Stardust sample return capsule (SRC) entered the atmosphere at a velocity of $12.6 \mathrm{~km} / \mathrm{s}$. This is the highest-energy vehicle entry ever undertaken. Shortly after entering the atmosphere, the Stardust SRC landed in Utah, allowing its precious scientific payload to be safely recovered.

The forebody thermal protection material used on Stardust was PICA (phenolic impregnated carbon ablator), which has also been selected as a candidate material for use on NASA's Orion crew exploration vehicle (CEV). The combination of having an entry velocity similar to Mars return and the use of PICA as heat shield material make it of significant interest to perform detailed analyses of the Stardust SRC entry even after the successful completion of its mission.

A number of studies of the aerothermodynamics of the Stardust SRC were performed before the mission [1-4]. These studies conducted flowfield analyses using both continuum computational fluid dynamics (CFD) methods (by solving the Navier-Stokes equations) and particle methods [using the direct simulation Monte Carlo method (DSMC)] [5] . However, the DSMC study [3] was focused on the high-altitude aerodynamics of the capsule and did not include the effects of ionization reactions. A more recent study

Presented as Paper 2007-4543 at the AIAA Thermophysics Conference, Miami, FL, 25-27 June 2007; received 29 February 2008; accepted for publication 7 October 2009. This material is declared a work of the U.S. Government and is not subject to copyright protection in the United States. Copies of this paper may be made for personal or internal use, on condition that the copier pay the $\$ 10.00$ per-copy fee to the Copyright Clearance Center, Inc., 222 Rosewood Drive, Danvers, MA 01923; include the code 0022-4650/ 10 and $\$ 10.00$ in correspondence with the CCC.

*Professor, Department of Aerospace Engineering, 1320 Beal Avenue. Associate Fellow AIAA.

'Research Scientist, Reacting Flow Environments Branch. Member AIAA.

${ }^{\ddagger}$ Senior Research Scientist, Reacting Flow Environments Branch, Mail Stop 230-2. Senior Member AIAA. compared CFD and DSMC simulation results for Stardust at $80 \mathrm{~km}$ [6].

The primary goal of this study is to perform high-altitude analyses of the aerothermodynamic environment on the forebody of the Stardust SRC. Computations are performed with both continuum and particle methods. Direct comparisons between the solutions provided by these techniques allow evaluation of differences between these approaches for modeling the strongly nonequilibrium thermochemical phenomena generated under highly energetic entry conditions. Comparisons in this environment are also needed to aid in further development of CFD-DSMC hybrid methods [7] to be extended to flows involving thermochemical nonequilibrium.

The geometry of the Stardust SRC and the entry conditions chosen for study are first described. The two numerical methods employed in the study are described in some detail. These consist of a CFD code for solving the Navier-Stokes equations with coupled nonequilibrium thermochemistry and a DSMC code that also includes thermochemical nonequilibrium. Emphasis is given on any differences in the ways in which the important thermochemical nonequilibrium processes are simulated. Results are then presented in which direct comparisons are made between several sets of continuum and particle simulations. The results are discussed and final conclusions are formulated.

\section{Flow Conditions}

The geometry of the Stardust SRC is shown schematically in Fig. 1. The forebody consists of a 60 deg half-angle, spherically blunted cone with a nose radius of $0.2202 \mathrm{~m}$. In the computations, a small portion of the shoulder is included that has a radius of $0.02 \mathrm{~m}$. The computations in this study are conducted for the Stardust entry conditions at 81 and $71 \mathrm{~km}$. These flow conditions are chosen due to the availability of detailed spectral measurements taken during the Stardust entry. The flowfield solutions generated in this study are used in a companion paper to compute spectra that are compared to the flight data [8]. The flow conditions are provided in Table 1 . The Knudsen numbers lie in a region where relatively small differences between continuum and particle simulation results for hypersonic flow are expected. For example, in comparing Mach 25 flow of 


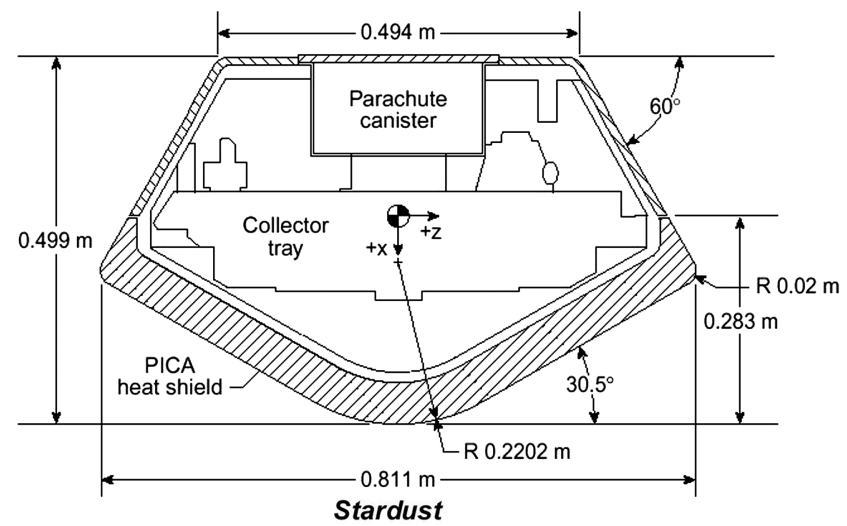

Fig. 1 Geometry of the Stardust return capsule.

nonreacting argon over a cylinder, differences in predicted peak heating between continuum and particle simulations were about $5 \%$ at $K n=0.01$ and about $2 \%$ at $K n=0.002$ [9]

\section{Numerical Methods}

The two different numerical approaches applied in this study are 1) CFD solution of the continuum Navier-Stokes equations using the DPLR code and 2) particle-based DSMC computation. In each case, an 11-species, 19-reaction, thermochemical nonequilibrium approach is adopted. The wall temperature is assumed to be fixed at a temperature of $2000 \mathrm{~K}$. The wall material PICA is assumed to be fully

Table 1 Freestream and surface parameters

\begin{tabular}{lcc}
\hline & \multicolumn{2}{c}{ Altitude, $\mathrm{km}$} \\
\cline { 2 - 3 } Parameter & 81 & 71 \\
\hline Freestream temperature, $\mathrm{K}$ & 217.6 & 221.6 \\
Freestream number density, molecules $/ \mathrm{m}^{3}$ & $2.643 \times 10^{20}$ & $1.156 \times 10^{21}$ \\
Freestream velocity, m/s & 12,385 & 12,063 \\
Freestream $\mathrm{O}_{2}$ mole fraction, $\%$ & 23.67 & 23.67 \\
Freestream $\mathrm{N}_{2}$ mole fraction, $\%$ & 76.23 & 76.23 \\
Freestream Knudsen number & 0.005 & 0.001 \\
Surface temperature, $\mathrm{K}$ & 2000 & 2700 \\
\hline \hline
\end{tabular}

catalytic to ions (that recombine into their neutral atoms and molecules) and atoms (that recombine into molecules). Ablation processes are not included in these studies.

\section{A. Details of the Continuum Flow Mode}

The continuum computations are performed using the DPLR CFD code $[10,11]$. DPLR is a parallel multiblock finite volume code that solves the Navier-Stokes equations including finite-rate chemistry and the effects of thermal nonequilibrium. The Euler fluxes are computed using a modified (low-dissipation) form of StegerWarming flux vector splitting [12], with third-order spatial accuracy obtained via MUSCL extrapolation [13]. Viscous fluxes are computed to second-order accuracy using a central-difference approach. DPLR has been used previously on several other planetary entry simulations $[10,14,15]$.

The flow is assumed to be in a state of thermochemical nonequilibrium with three separate temperatures for the translational, rotational, and vibrational modes. Viscous transport and thermal conductivity are modeled using the mixing rules presented by Gupta et al. [16], which have been shown to be reasonable approximations of the more accurate Chapman-Enskog relations in this flow regime $[17,18]$. The bifurcation method is used to compute the species diffusion coefficients [19]. This method allows for the variations in species diffusion coefficients to be accurately modeled without sacrificing the requirement that the diffusion velocities sum identically to zero.

Rotational relaxation is modeled using the relaxation time based on the Parker model [20]. Vibrational relaxation is modeled using a Landau-Teller formulation, where relaxation times are obtained from Millikan and White [21], assuming simple harmonic oscillators, with the high-temperature correction of Park [22].

The baseline DPLR case employs the rates in Table $\underline{2}$, the standard Park [22] two-temperature model for dissociation, in which the controlling temperature $T=T_{t}^{0.5} \times T_{v}^{0.5}$, and all other reactions are controlled solely by the translational temperature. This baseline DPLR model was established by Olynick et al. [1] and applied to analyze the aerothermodynamics of Stardust before its launch. The baseline 19-reaction finite-rate air-chemistry model uses the rates $[23,24]$ that are listed in Table 2 . The backward rates are computed using the forward rates, and equilibrium constants are evaluated using the van't Hoff equation.

Table 2 Baseline forward reaction rate coefficients $\left(\mathrm{m}^{3} / \mathrm{molecule} / \mathrm{s}\right)$

\begin{tabular}{|c|c|c|}
\hline Number & Reaction & Rate coefficient \\
\hline $1 \mathrm{M}$ & $\mathrm{N}_{2}+\mathrm{M} \Leftrightarrow \mathrm{N}+\mathrm{N}+\mathrm{M}$ & $1.162 \times 10^{-8} \mathrm{~T}^{-1.6} \exp (-113,200 / \mathrm{T})$ \\
\hline $1 \mathrm{~A}$ & $\mathrm{~N}_{2}+\mathrm{A} \Leftrightarrow \mathrm{N}+\mathrm{N}+\mathrm{A}$ & $4.980 \times 10^{-8} \mathrm{~T}^{-1.6} \exp (-113,200 / \mathrm{T})$ \\
\hline $1 \mathrm{E}$ & $\mathrm{N}_{2}+\mathrm{E}^{-} \Leftrightarrow \mathrm{N}+\mathrm{N}+\mathrm{A}$ & $4.980 \times 10^{-6} \mathrm{~T}^{-1.6} \exp (-113,200 / \mathrm{T})$ \\
\hline $2 \mathrm{M}$ & $\mathrm{O}_{2}+\mathrm{M} \Leftrightarrow \mathrm{O}+\mathrm{O}+\mathrm{M}$ & $3.321 \times 10^{-9} \mathrm{~T}^{-1.5} \exp (-59,400 / \mathrm{T})$ \\
\hline $2 \mathrm{~A}$ & $\mathrm{O}_{2}+\mathrm{A} \Leftrightarrow \mathrm{O}+\mathrm{O}+\mathrm{A}$ & $1.660 \times 10^{-8} \mathrm{~T}^{-1.5} \exp (-59,400 / \mathrm{T})$ \\
\hline $3 \mathrm{M}$ & $\mathrm{NO}+\mathrm{M} \Leftrightarrow \mathrm{N}+\mathrm{O}+\mathrm{M}$ & $8.302 \times 10^{-15} \exp (-75,500 / \mathrm{T})$ \\
\hline $3 \mathrm{~A}$ & $\mathrm{NO}+\mathrm{A} \Leftrightarrow \mathrm{N}+\mathrm{O}+\mathrm{A}$ & $1.826 \times 10^{-13} \exp (-75,500 / \mathrm{T})$ \\
\hline 4 & $\mathrm{O}+\mathrm{NO} \Leftrightarrow \mathrm{N}+\mathrm{O}_{2}$ & $1.389 \times 10^{-17} \exp (-19,700 / \mathrm{T})$ \\
\hline 5 & $\mathrm{O}+\mathrm{N}_{2} \Leftrightarrow \mathrm{N}+\mathrm{NO}$ & $1.069 \times 10^{-12} \mathrm{~T}^{-1.000} \exp (-37,500 / \mathrm{T})$ \\
\hline 6 & $\mathrm{~N}+\mathrm{N} \Leftrightarrow \mathrm{N}_{2}^{+}+\mathrm{E}^{-}$ & $3.387 \times 10^{-17} \exp (-67,700 / \mathrm{T})$ \\
\hline 7 & $\mathrm{O}+\mathrm{O} \Leftrightarrow \mathrm{O}_{2}^{+}+\mathrm{E}^{-}$ & $1.859 \times 10^{-17} \exp (-81,200 / \mathrm{T})$ \\
\hline 8 & $\mathrm{~N}+\mathrm{O} \Leftrightarrow \mathrm{NO}^{+}+\mathrm{E}^{-}$ & $8.766 \times 10^{-18} \exp (-32,000 / \mathrm{T})$ \\
\hline $9 \mathrm{a}$ & $\mathrm{N}+\mathrm{E}^{-} \Leftrightarrow \mathrm{N}^{+}+2 \mathrm{E}^{-}$ & $4.151 \times 10^{4} \mathrm{~T}^{-3.82} \exp (-168,600 / \mathrm{T})$ \\
\hline $9 \mathrm{~b}$ & $\mathrm{~N}+\mathrm{E}^{-} \Leftrightarrow \mathrm{N}^{+}+2 \mathrm{E}^{-}$ & $8.434 \times 10^{-14} \exp (-121,000 / \mathrm{T})$ \\
\hline $10 \mathrm{a}$ & $\mathrm{O}+\mathrm{E}^{-} \Leftrightarrow \mathrm{O}^{+}+2 \mathrm{E}^{-}$ & $6.475 \times 10^{3} \mathrm{~T}^{-3.78} \exp (-158,500 / \mathrm{T})$ \\
\hline $10 \mathrm{~b}$ & $\mathrm{O}+\mathrm{E}^{-} \Leftrightarrow \mathrm{O}^{+}+2 \mathrm{E}^{-}$ & $1.054 \times 10^{-14} \exp (-106,200 / \mathrm{T})$ \\
\hline 11 & $\mathrm{~N}_{2}+\mathrm{O}^{+} \Leftrightarrow \mathrm{O}+\mathrm{N}_{2}^{+}$ & $1.511 \times 10^{-18} \mathrm{~T}^{0.360} \exp (-22,800 / \mathrm{T})$ \\
\hline 12 & $\mathrm{NO}+\mathrm{O}^{+} \Leftrightarrow \mathrm{O}_{2}+\mathrm{N}^{+}$ & $2.324 \times 10^{-25} \mathrm{~T}^{1.900} \exp (-15,300 / \mathrm{T})$ \\
\hline 13 & $\mathrm{O}_{2}+\mathrm{NO}^{+} \Leftrightarrow \mathrm{NO}+\mathrm{O}_{2}^{+}$ & $3.985 \times 10^{-17} \mathrm{~T}^{0.410} \exp (-32,600 / \mathrm{T})$ \\
\hline 14 & $\mathrm{~N}+\mathrm{NO}^{+} \Leftrightarrow \mathrm{O}+\mathrm{N}_{2}^{+}$ & $1.195 \times 10^{-16} \exp (-35,500 / \mathrm{T})$ \\
\hline 15 & $\mathrm{O}+\mathrm{NO}^{+} \Leftrightarrow \mathrm{O}_{2}+\mathrm{N}^{+}$ & $1.660 \times 10^{-18} \mathrm{~T}^{0.500} \exp (-77,200 / \mathrm{T})$ \\
\hline 16 & $\mathrm{~N}+\mathrm{O}_{2}^{+} \Leftrightarrow \mathrm{O}_{2}+\mathrm{N}^{+}$ & $1.444 \times 10^{-16} \mathrm{~T}^{0.140} \exp (-28,600 / \mathrm{T})$ \\
\hline 17 & $\mathrm{~N}_{2}+\mathrm{O}_{2}^{+} \Leftrightarrow \mathrm{O}_{2}+\mathrm{N}_{2}^{+}$ & $1.644 \times 10^{-17} \exp (-40,700 / \mathrm{T})$ \\
\hline 18 & $\mathrm{~N}+\mathrm{NO}^{+} \Leftrightarrow \mathrm{N}_{2}+\mathrm{O}^{+}$ & $5.645 \times 10^{-17} \mathrm{~T}^{-1.080} \exp (-12,800 / \mathrm{T})$ \\
\hline 19 & $\mathrm{O}+\mathrm{NO}^{+} \Leftrightarrow \mathrm{N}+\mathrm{O}_{2}^{+}$ & $1.195 \times 10^{-17} \mathrm{~T}^{0.290} \exp (-48,600 / \mathrm{T})$ \\
\hline
\end{tabular}


To address differences in chemistry modeling between DPLR and DSMC, use of different controlling temperatures $T=T_{t}^{n} \times T_{v}^{1-n}$ is considered for both dissociation and ionization reactions. For dissociation, heat-bath studies indicate that nonequilibrium DSMC dissociation rates are closer to those obtained in DPLR with $n=0.3$ rather than the baseline of $n=0.5$. For direct ionization, in DSMC the collision energy is determined by the relative velocity between the atom and the electron that is generally dominated by the electron velocity. Thus, DSMC simulation of direct ionization is more closely approximated in DPLR using $n=0.0$, assuming that the vibrational temperature in DPLR is a better approximation to the electron temperature than the translational temperature. In presentation of results, the baseline DPLR case is indicated by $b$ and the modifiedchemistry case is indicated by $\mathrm{m}$.

Surface catalysis is modeled using a diffusion-limited approach [22]. The surface is assumed to be fully catalytic to $\mathrm{N}_{2}$ and $\mathrm{O}_{2}$ recombination, which should be reasonable for the PICA thermal protection system (TPS) material, based on its expected similarity to graphite TPS systems.

\section{B. Details of the Particle Flow Model}

The particle computations employ a DSMC code developed specifically for hypersonic ionized flow simulations [25]. Models are implemented for rotational [26] and vibrational [27] energy exchange that are consistent with those employed in DPLR. Most of the chemical reactions are simulated using the total collision energy (TCE) model, except for the dissociation of nitrogen and oxygen. These reactions employ the vibrationally favored dissociation (VFD) model [28]. The VFD model makes it possible to bias the dissociation-reaction probability in favor of molecules that possess a higher vibrational energy. In this way, the important phenomenon of vibration-dissociation coupling can be simulated. In the present work, the VFD favoring parameter $\phi$ is set to 2.0 for nitrogen dissociation and 0.5 for oxygen dissociation, as determined in prior work [28]. A recent detailed DSMC study of nitrogen dissociation suggests that the VFD model with $\phi=2$ emulates the behavior of a more sophisticated approach [29].

The baseline set of chemical reactions and rate coefficients employed in the DSMC computations are listed in Table 1 . All of the forward rates are the same as those employed in DPLR, except for the two direct ionization reactions:

$$
\begin{aligned}
& \mathrm{N}+\mathrm{E}^{-} \rightarrow \mathrm{N}^{+}+2 \mathrm{E}^{-} \\
& \mathrm{O}+\mathrm{E}^{-} \rightarrow \mathrm{O}^{+}+2 \mathrm{E}^{-}
\end{aligned}
$$

It is not possible to employ the low negative-temperature exponents of the rates employed by DPLR in the DSMC TCE chemistry model. The rates employed by DSMC are those reported by Wilson [30] and are listed as reactions $9 \mathrm{~b}$ and $10 \mathrm{~b}$ in Table 2 . The lower activation energies in these rates are based on the premise that most atomic ionization occurs not from the ground electronic state, but rather from the lowest excited states of nitrogen and oxygen. These rates are also employed in some DPLR computations. The Park [23] and Wilson [30] ionization rates for both nitrogen and oxygen are compared in Fig. 2 as a function of temperature.

The backward steps of all chemical reactions are modeled using the approach described by Boyd [31] that provides consistency with the CFD approach.

The presence of electrons in the flowfield presents a challenge for the DSMC technique due to their very small mass and associated very high thermal velocities. Under the assumption of ambipolar diffusion, in which the diffusion rates of electrons and ions are assumed to be equal, the standard approach in DSMC for handling this situation is to tie each electron particle to the ion particle with which it was created in the ionization process [32]. In the present highly energetic flow condition, the ionization degree is expected to be significant, and keeping track of pairs of electrons and ions can cause difficulties for the standard DSMC approach. Therefore, a model previously developed for DSMC computation of plasma thrusters

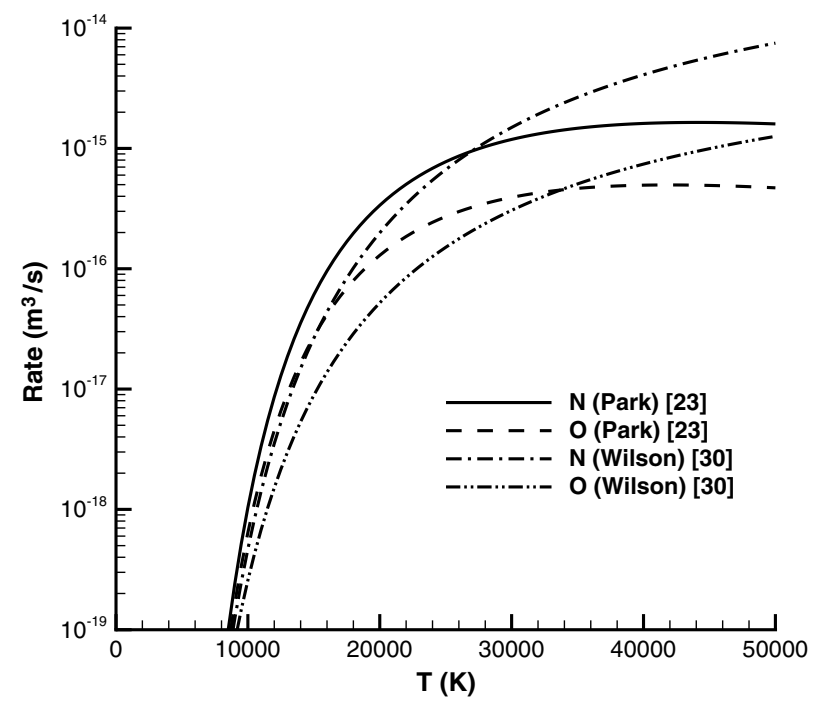

Fig. 2 Direct ionization rates of atomic nitrogen and oxygen as a function of temperature.

[33] is employed here. In this approach, the average ion velocity in each computational cell is evaluated and all electrons in each cell are moved with this average velocity. While the approach does not guarantee charge neutrality, in practice, it is found that neutrality is achieved everywhere in the flow domain within a few percent. This approach for handling electrons is significantly more robust than the standard DSMC approach.

The vehicle surface is treated as fully diffuse at the wall temperatures listed in Table 1 . The surface is fully catalytic to atoms that are recombined to molecules and to ions and electrons that are recombined to the neutral species.

\section{Results at 81 Kilometers}

The meshes used in the DPLR computations consist of three blocks containing $24 \times 128,64 \times 128$, and $48 \times 128$ cells. In each case, the mesh is grown out hyperbolically from the vehicle surface to ensure orthogonality and then adapted to contours of Mach number.

The DSMC computations employ a single mesh containing 100 by 200 cells, in which the cell dimension in the flow direction is everywhere less than the local mean free path. Typically, about two million particles are employed, of which 40,000 are electrons. The time step used in the DSMC computations is smaller than the mean free time for heavy particles anywhere in the flowfield. The smaller electron collision times are resolved by subcycling.

Figure $\underline{3}$ provides a comparison of the translational temperature contours obtained with DSMC (upper) and with the baseline DPLR computation (indicated in subsequent plots as $b$, and the DPLR case with modified-chemistry modeling is indicated as $\mathrm{m}$ ). Qualitatively, the comparison of temperature contours shows the following:

1) The shock wave predicted by DSMC is significantly thicker than that simulated by DPLR.

2) The peak translational temperature from DSMC is significantly higher than that simulated by DPLR.

Quantitative differences between the simulation results can be assessed using Fig. 4, which shows translational temperature profiles along the stagnation streamline. In all comparisons, the solid lines represent the baseline DPLR results, the dashed lines are the modified DPLR results, and the dash-dot-dash lines are the DSMC results. Also note that in all subsequent plots, the stagnation point is located at $Z=0 \mathrm{~m}$. These profiles show enormous differences between the DSMC and DPLR solutions that were not anticipated based on prior nonreacting CFD/DSMC comparisons at Mach 25 [9]. DSMC predicts a peak translational temperature that is more than a factor of 2 higher than the baseline DPLR result. The modified DPLR result lies considerably closer to DSMC, but significant differences remain. 


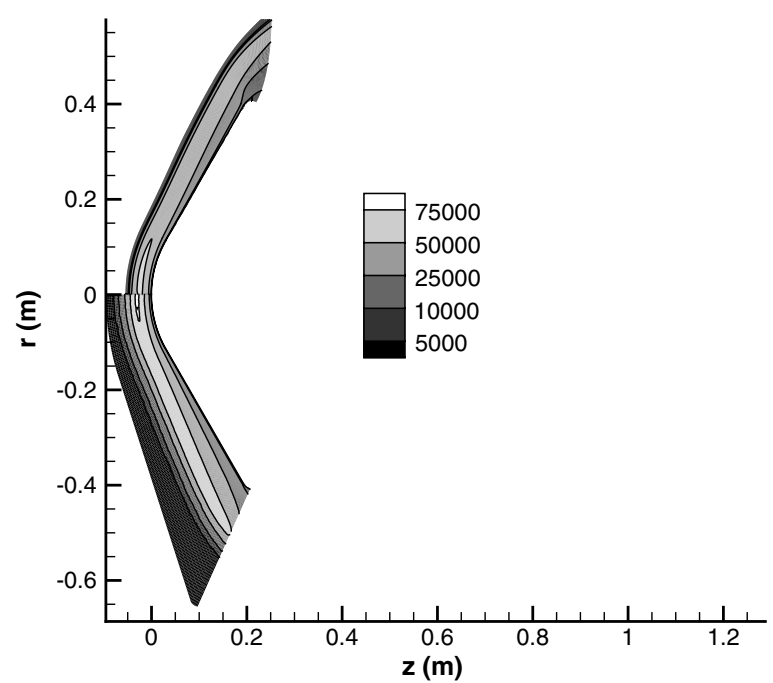

Fig. 3 Contours of translational temperature $(K)$ for the Stardust return capsule at $81 \mathrm{~km}$ obtained using DSMC (lower) and baseline DPLR (upper).

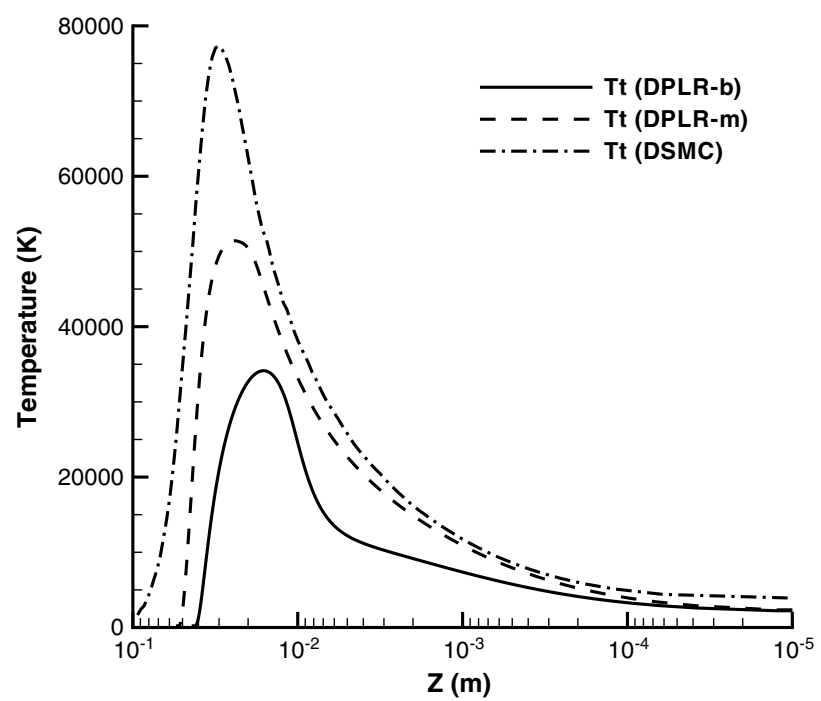

Fig. 4 Profiles of translational temperature along the stagnation streamline at $81 \mathrm{~km}$.

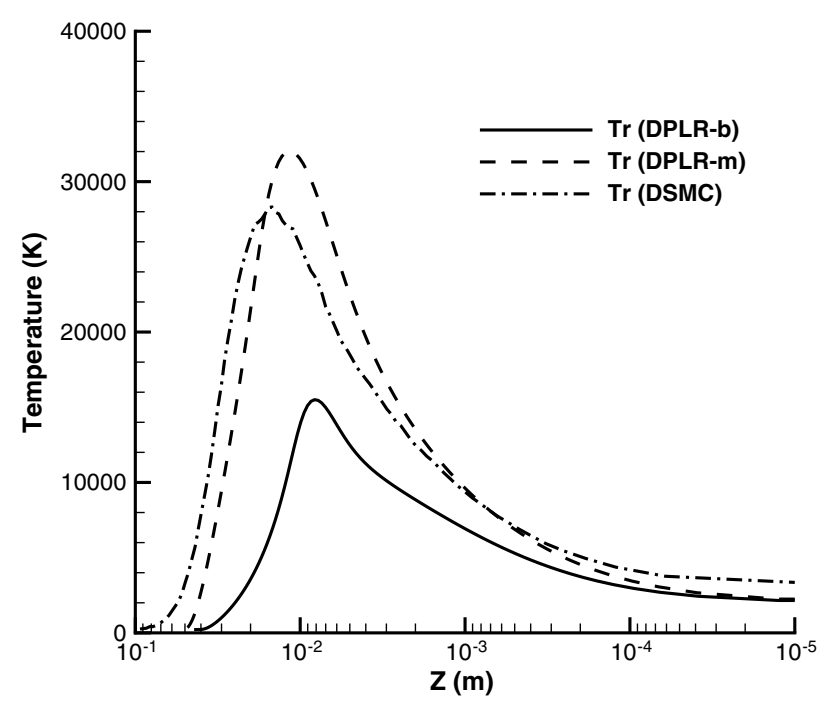

Fig. 5 Profiles of rotational temperature along the stagnation streamline at $81 \mathrm{~km}$.

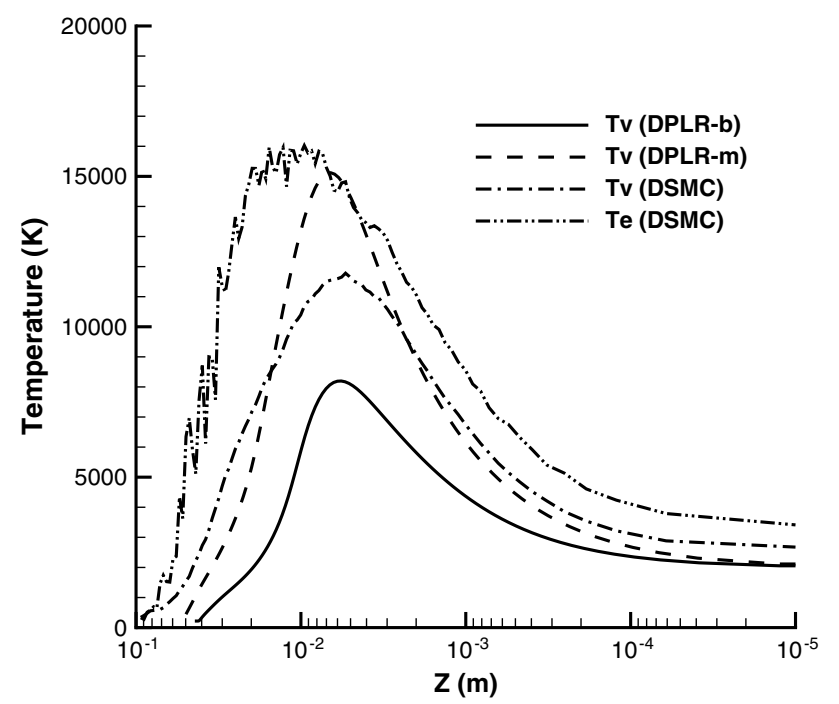

Fig. 6 Profiles of vibrational and electron temperature along the stagnation streamline at $81 \mathrm{~km}$.

Profiles for rotational temperature are shown in Fig. 5. Once again, the modified DPLR and DSMC results show better correspondence than the baseline DPLR results. Profiles for vibrational temperature are shown in Fig. 6. Once again, the baseline DPLR result is significantly lower than the other two. The peak vibrational temperature from the modified DPLR simulation is a little higher than the peak from DSMC. Also included in Fig. 6 is the profile of electron temperature from the DSMC computation. Whereas DSMC provides the translational temperature of the electrons, DPLR assumes that the electrons have the same translational temperature as the bulk gas. In terms of radiation modeling, it is a common assumption to use the vibrational temperature from CFD calculations to represent the electron temperature responsible for activation processes. The electron temperature is consistently higher than the DSMC vibrational temperature, generally higher than the modified DPLR vibrational temperature, and significantly higher than the baseline DPLR vibrational temperature.

Profiles of the number densities of molecular nitrogen are shown in Fig. 7. The shock standoff distances predicted by the modified DPLR and DSMC simulations are in good agreement, whereas the baseline DPLR computation predicts a significantly smaller standoff distance. The number density profiles of atomic nitrogen and oxygen are shown in Figs. $\underline{8}$ and $\underline{9}$, respectively. In all of these profiles of neu-

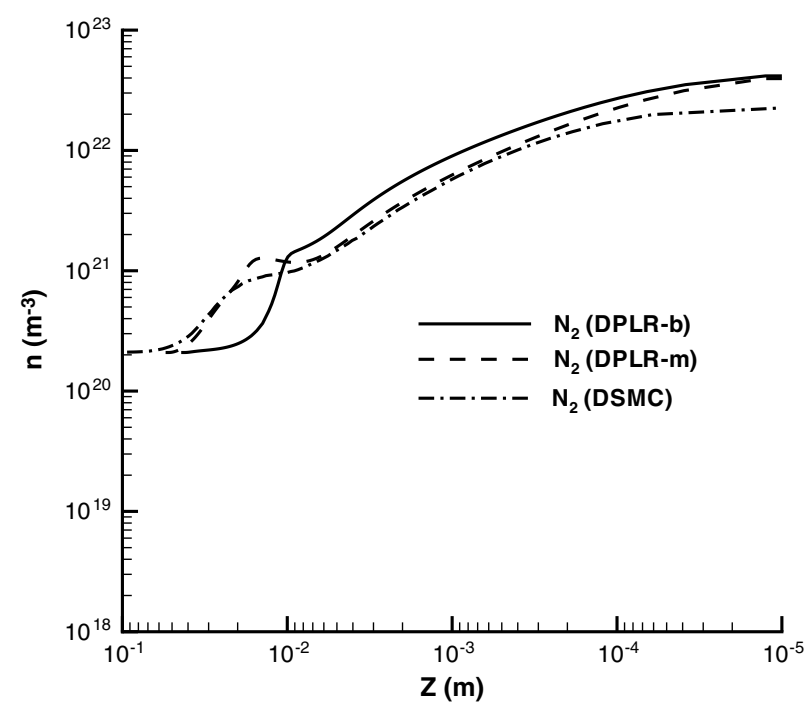

Fig. 7 Profiles of $N_{2}$ number density along the stagnation streamline at $81 \mathrm{~km}$. 


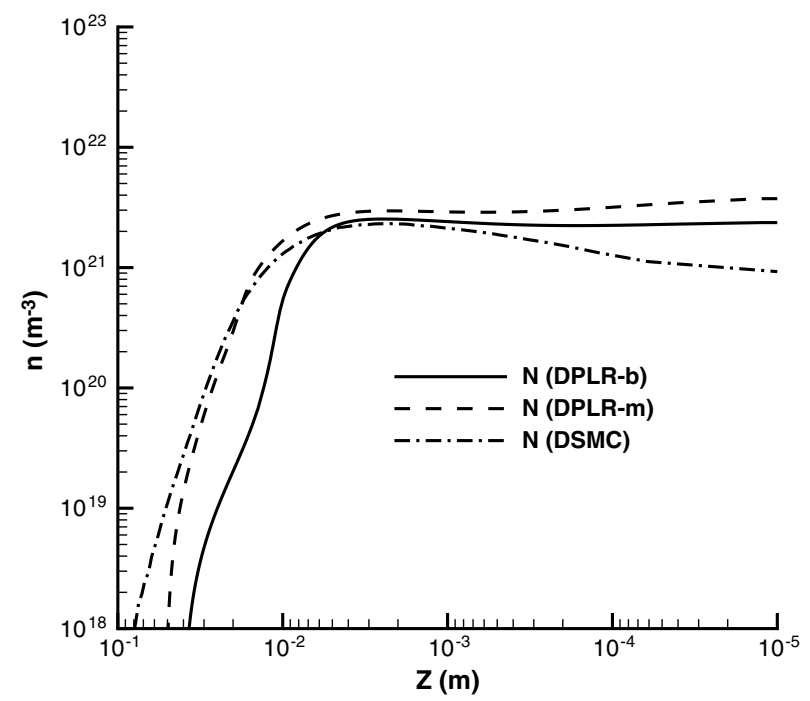

Fig. 8 Profiles of $\mathrm{N}$ number density along the stagnation streamline at $81 \mathrm{~km}$.

tral number densities, the DSMC and modified DPLR simulations agree well in the shock-front and postshock regions, and then DSMC predicts lower values near the vehicle surface.

Profiles of the number densities of molecular nitrogen ions are shown in Fig. 10. Here, there is significant disagreement between the two DPLR cases, with the baseline chemistry predicting orders-ofmagnitude-lower levels of $\mathrm{N}_{2}^{+}$. The DSMC and modified DPLR profiles show much better agreement with one another. Profiles of the atomic ion number density are shown in Fig. 11. Here, again, there are significant differences between the two DPLR solutions, with the modified case producing significantly less ionization that is in much better agreement with the DSMC profiles. The baseline DPLR solution predicts a peak $\mathrm{N}^{+}$number density that is about a factor of 25-30 higher than the peak predictions from the other two simulations.

Profiles of the capsule surface pressure are compared in Fig. 12. As expected, all three profiles are in very good agreement. The surface heat flux profiles are compared in Fig. 13. The peak heat fluxes from the baseline and modified DPLR simulations are 15 and $40 \%$ higher than the peak DSMC heat flux, respectively. The better agreement achieved between the baseline DPLR solution and DSMC for heat flux is considered fortuitous and results in part from the baseline DPLR solution producing a lower temperature gradient in the

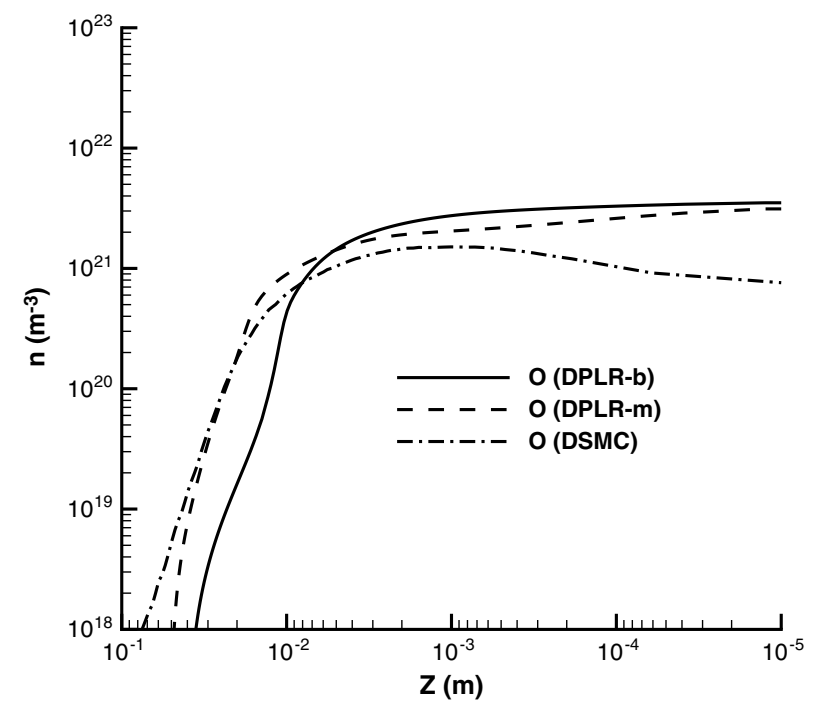

Fig. 9 Profiles of $\mathrm{O}$ number density along the stagnation streamline at $81 \mathrm{~km}$.

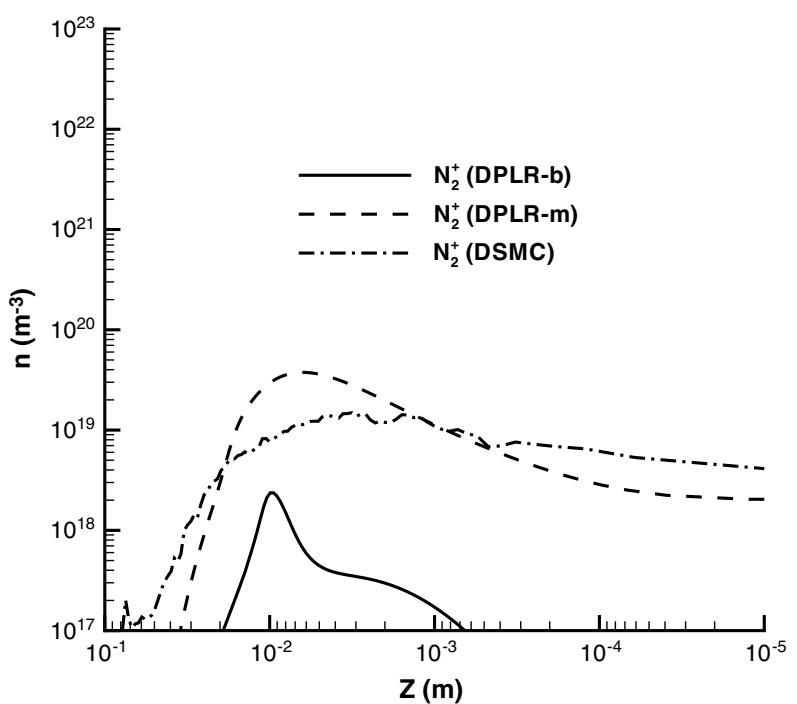

Fig. 10 Profiles of $\mathrm{N}_{2}^{+}$number density along the stagnation streamline at $81 \mathrm{~km}$.

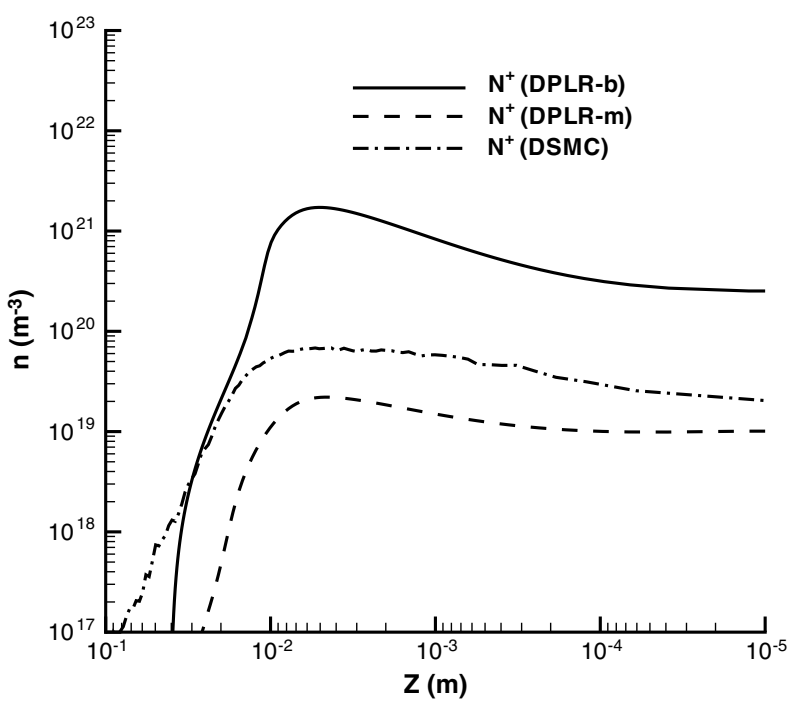

Fig. 11 Profiles of $\mathrm{N}^{+}$number density along the stagnation streamline at $81 \mathrm{~km}$.

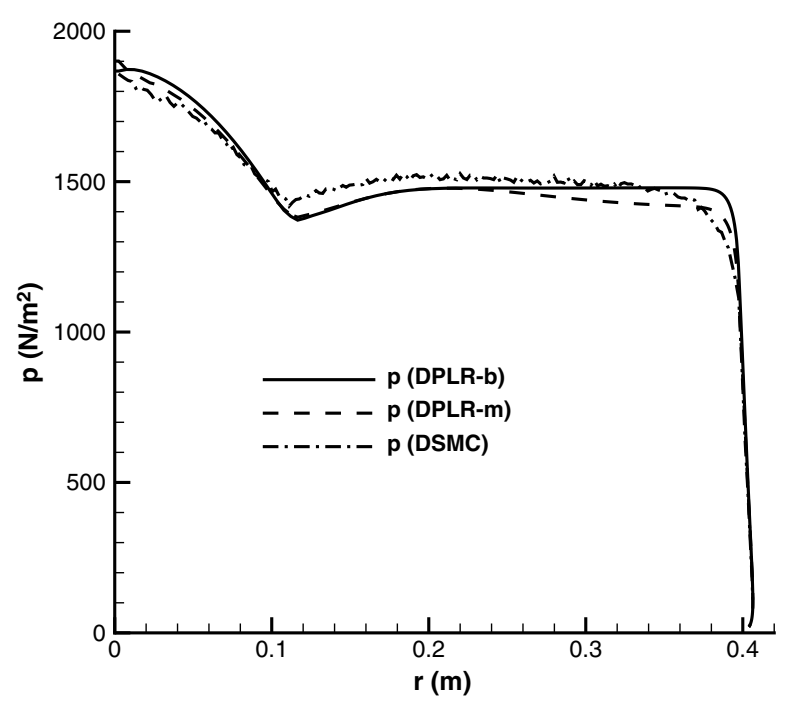

Fig. 12 Profiles of pressure along the surface of the Stardust return capsule at $81 \mathrm{~km}$. 


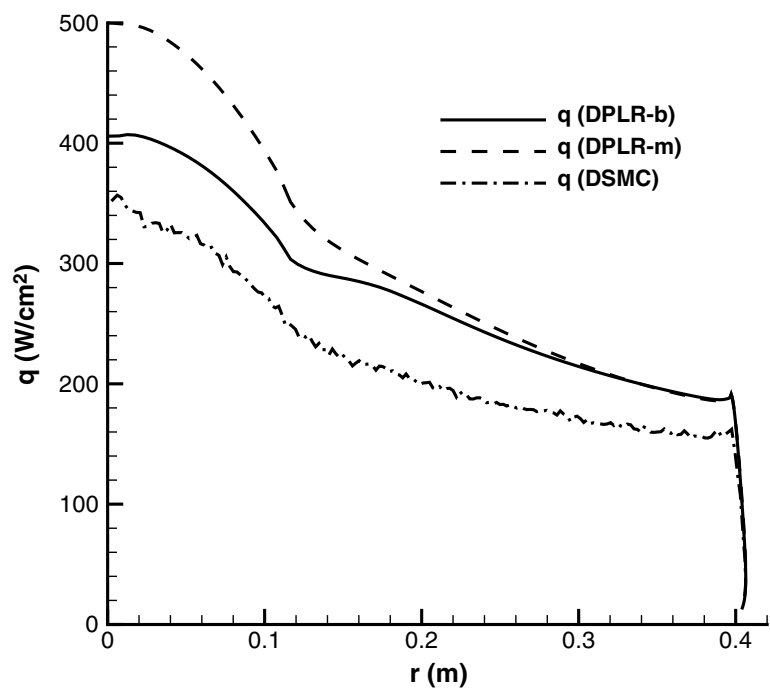

Fig. 13 Profiles of heat flux along the surface of the Stardust return capsule at $81 \mathrm{~km}$.

postshock region in comparison to the modified DPLR solution. For most flowfield quantities, the modified DPLR solution gives better correspondence to the DSMC results.

The levels of disagreement between DSMC and the two DPLR solutions are, at first glance, surprising for a flow with an overall Knudsen number of 0.005 . Because of the significant effects on the flowfield of the different chemistry models, the most useful comparison to consider is between DSMC and the modified DPLR result. The solution differences in the vicinity of the shock are not unexpected and arise from the failure of the continuum approach to simulate the strongly-non-Maxwellian velocity distribution functions that are generated there. The strong noncontinuum aspect of the flow is illustrated in Fig. 14, in which profiles are shown along the stagnation streamline of the gradient-length local Knudsen number [34] obtained from the two DPLR solutions. Here, the characteristic length scale employed to evaluate $K n_{\mathrm{GLL}}$ is the normalized temperature gradient. Careful comparisons between DSMC and CFD under hypersonic flow conditions showed that the Navier-Stokes equations are inaccurate whenever $K n_{\mathrm{GLL}}>0.05$ [34]. For the present flow, very large values of $K n_{\mathrm{GLL}}$ are obtained in the shock, as expected. However, large values are also obtained in the relatively cold boundary layer, due to the significant temperature gradients experienced there.

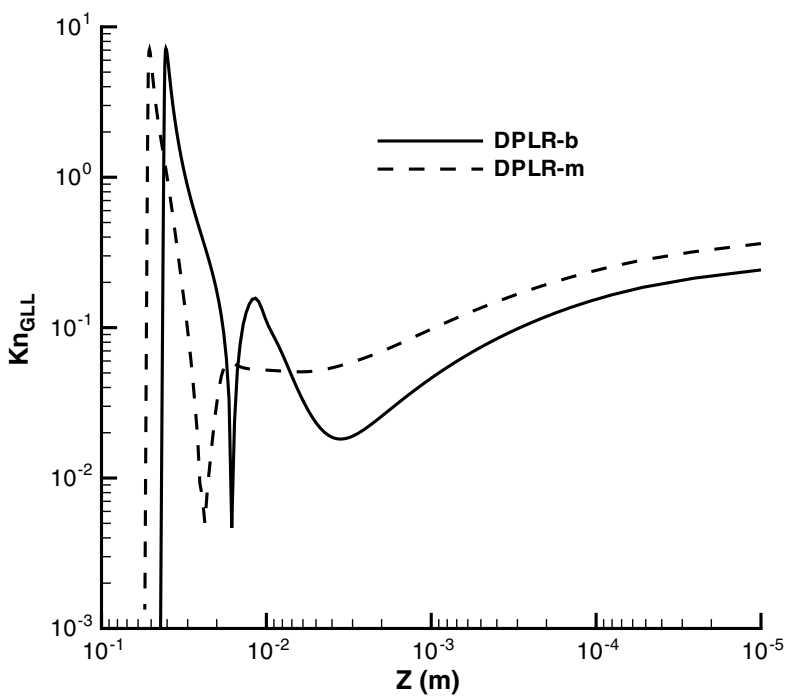

Fig. 14 Profiles of gradient-length local Knudsen number along the stagnation streamline obtained from the two DPLR solutions at $81 \mathrm{~km}$.

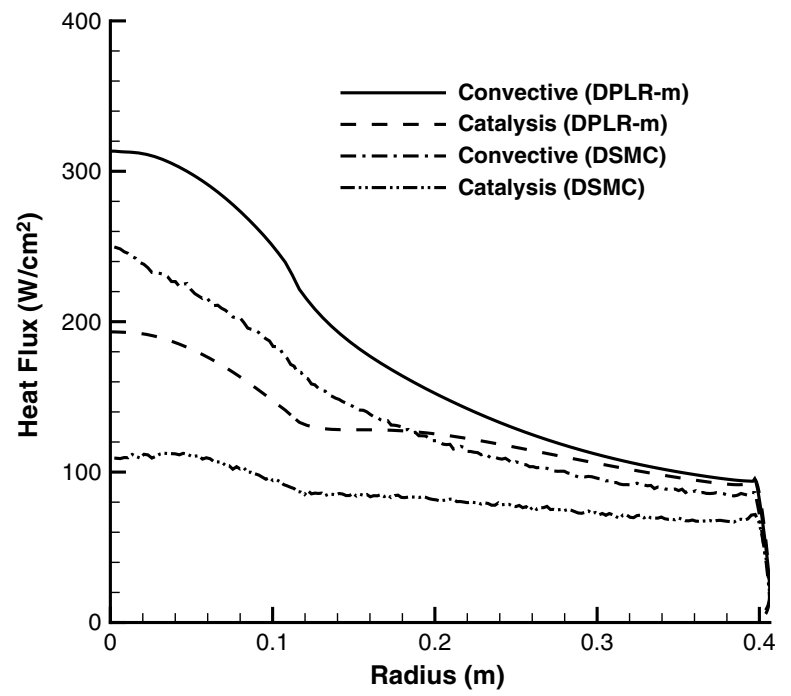

Fig. 15 Profiles of the contributions to heat flux along the surface of the Stardust return capsule at $81 \mathrm{~km}$.

Detailed analysis of the heat flux is provided in Fig. 15, in which the convective and surface catalysis contributions to the heating are shown from the modified DPLR and the DSMC solutions. The DSMC peak convective heating is about $25 \%$ less than the DPLR result. In prior studies of Mach 25 flow of argon over a cylinder, CFD gave peak heat transfer rates that were $15 \%$ higher than DSMC for a stagnation-point value of about $K n_{\mathrm{GLL}}=0.5$ [9]. That is approximately the value obtained here from the modified DPLR solution. Hence, it should not be so surprising to find significant differences in peak convective heating between DPLR and DSMC solutions under these flow conditions.

A much more significant difference is shown in Fig. 15 for the catalytic heating, in which the peak DSMC result is about a factor of 2 lower than the DPLR value. Most of the catalytic heating arises from recombination of atomic nitrogen and oxygen, and it is clear from Figs. 8 and 9 that DSMC predicts about a factor-of-2-smaller densities for these atoms at the stagnation point. This behavior is also related to the large value of $K n_{\mathrm{GLL}}$ at the surface, which explains why the DSMC temperatures are higher than the DPLR values as the surface is approached. Even with fully diffuse reflection, DSMC will produce a temperature jump under conditions of high local Knudsen number. The higher DSMC translational temperature at the surface leads to a corresponding lower total number density, since the pressure is relatively insensitive to nonequilibrium effects.

\section{Results at 71 Kilometers}

The meshes used in the DPLR computations again consist of three blocks containing $24 \times 128,64 \times 128$, and $48 \times 128$ cells. The DSMC computations employ a single mesh containing 200 by 200 cells, in which the cell dimension in the flow direction is everywhere less than the local mean free path. Typically, about three million particles are employed, of which 100,000 are electrons. The time step used in the DSMC computations is smaller than the mean free time anywhere in the flowfield.

Translational temperature profiles along the stagnation streamline are shown in Fig. 16. Significant differences remain between DSMC and the baseline $\overline{D P} L R$ calculation. Although the modified DPLR result shows a higher peak value than the baseline DPLR case, the two simulations are identical in the postshock region. The DSMC solution provides higher temperatures throughout the boundary layer.

Profiles for vibrational temperature and the electron temperature from DSMC are shown in Fig. 17. The modified DPLR profile has a higher peak than the baseline DPLR case, but the two simulations again agree in the postshock region. The DSMC results show good agreement in the postshock region between the vibrational and 


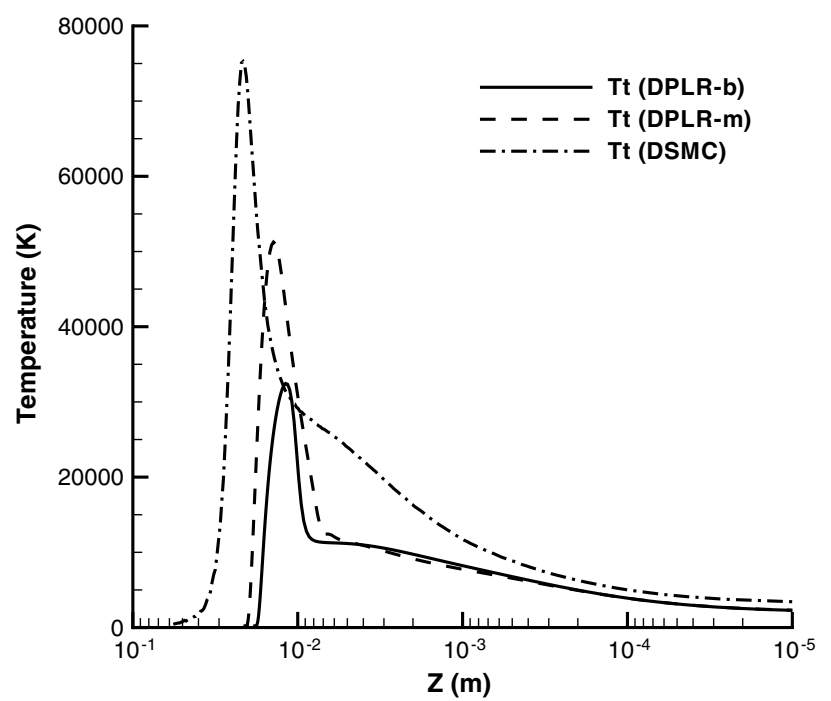

Fig. 16 Profiles of translational temperature along the stagnation streamline at $71 \mathrm{~km}$.

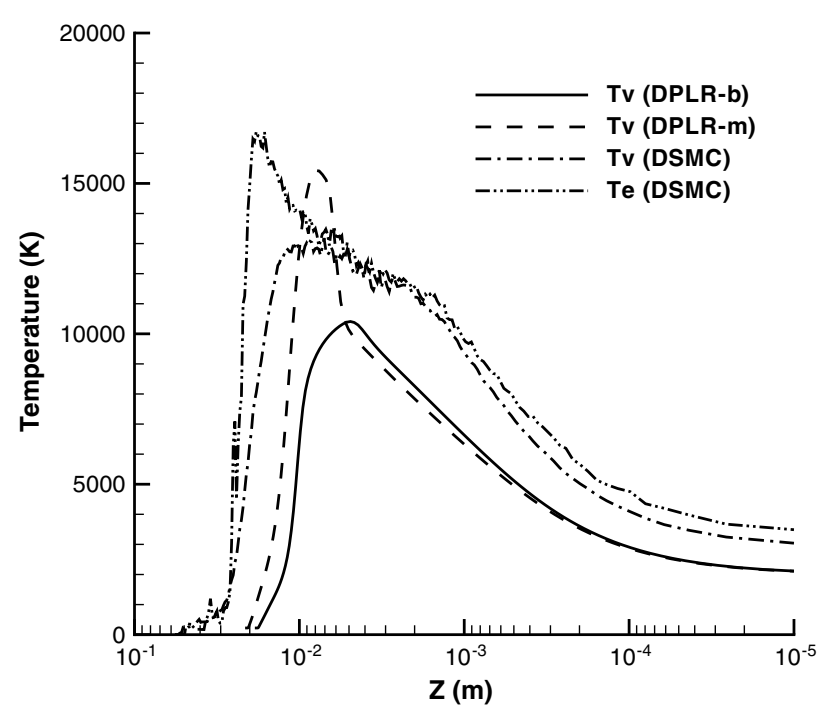

Fig. 17 Profiles of vibrational and electron temperature along the stagnation streamline at $71 \mathrm{~km}$.

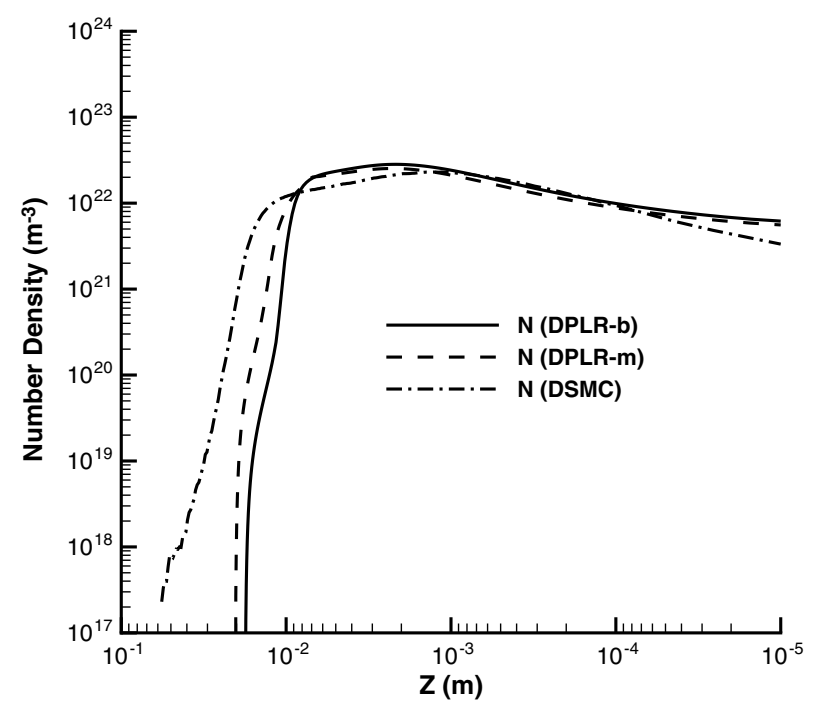

Fig. 18 Profiles of $\mathrm{N}$ number density along the stagnation streamline at $71 \mathrm{~km}$.

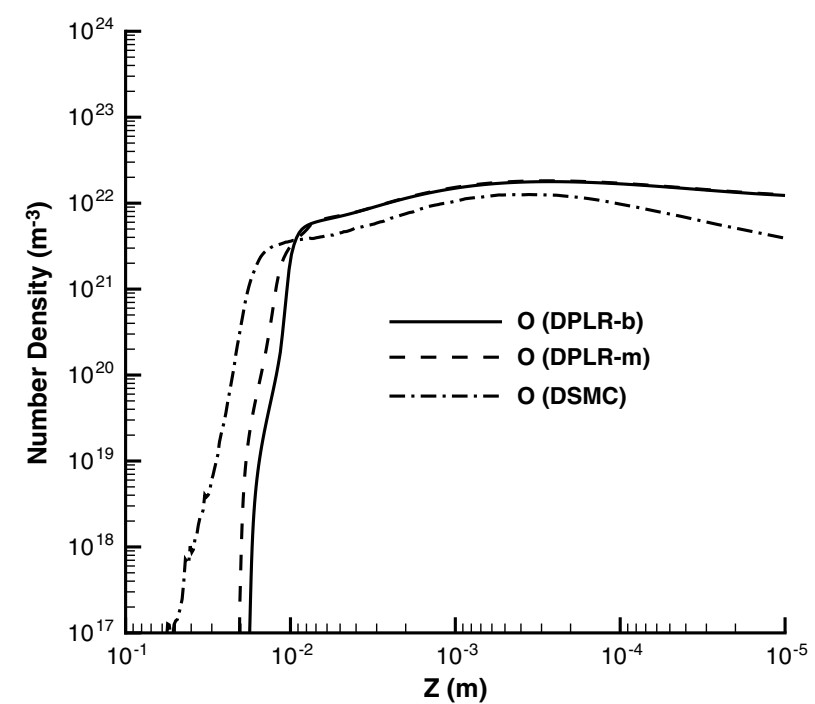

Fig. 19 Profiles of $\mathrm{O}$ number density along the stagnation streamline at $71 \mathrm{~km}$.

electron temperatures, but the values are consistently higher than the DPLR profiles.

The number density profiles of atomic nitrogen and oxygen are shown in Figs. 18 and 19, respectively. For nitrogen, there is excellent agreement between the three profiles. For oxygen, there is a slight reduction in the degree of dissociation predicted by DSMC. Similar to the $81 \mathrm{~km}$ case, although to a lesser extent, the higher DSMC temperatures near the stagnation point produce lower number densities.

The number density profiles of molecular nitrogen ions are shown in Fig. 20. The DSMC profile is at a higher level throughout. The modified DPLR case has a higher peak than the baseline DPLR case, but in the postshock region the two solutions are in good agreement. Profiles of the atomic ion number density are shown in Fig. 21. In this case, the two DPLR profiles are in good agreement and for most of the shock layer show significantly higher levels than the DSMC case. Note that the density of $\mathrm{N}^{+}$is at least an order of magnitude higher than for $\mathrm{N}_{2}^{+}$, and so the electron number density comparisons essentially follow the trends shown in Fig. 21.

Profiles of the surface pressure compared in Fig. 22 again show good agreement between the three cases. The profiles of surface heat flux shown in Fig. 23 indicate good agreement between the two DPLR cases, whereas the DSMC profile is consistently about $15 \%$

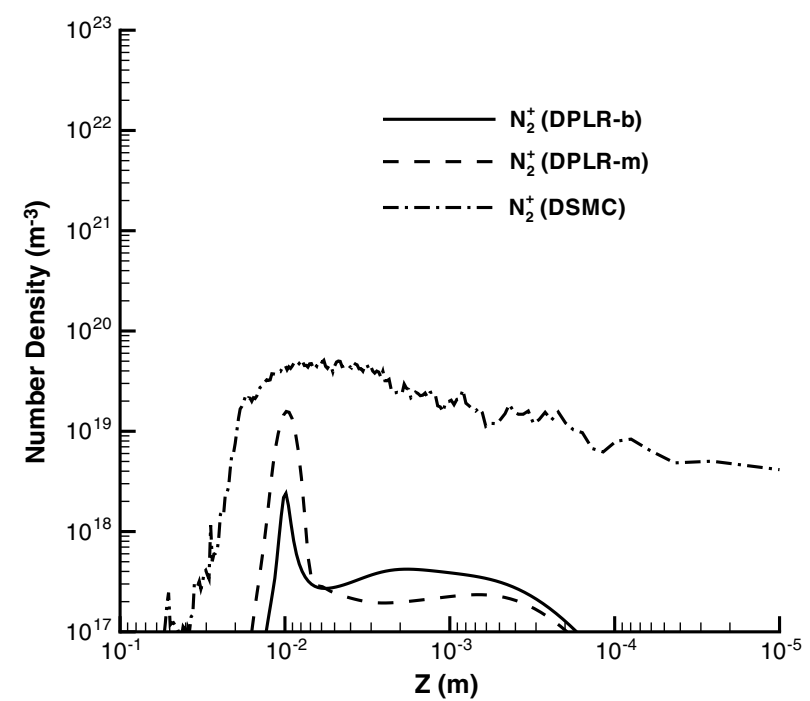

Fig. 20 Profiles of $\mathrm{N}_{2}^{+}$number density along the stagnation streamline at $71 \mathrm{~km}$. 


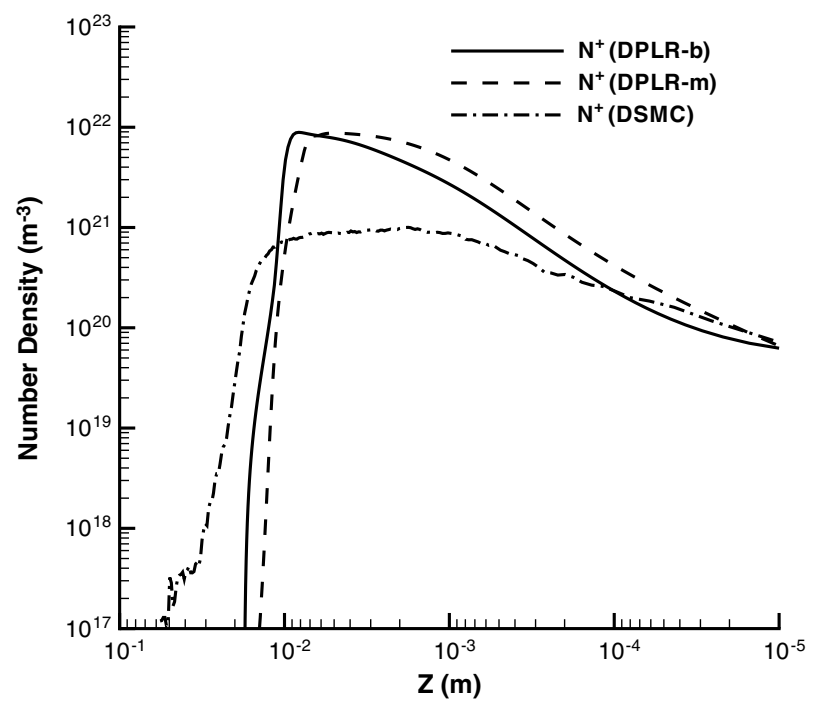

Fig. 21 Profiles of $\mathrm{N}^{+}$number density along the stagnation streamline at $71 \mathrm{~km}$.

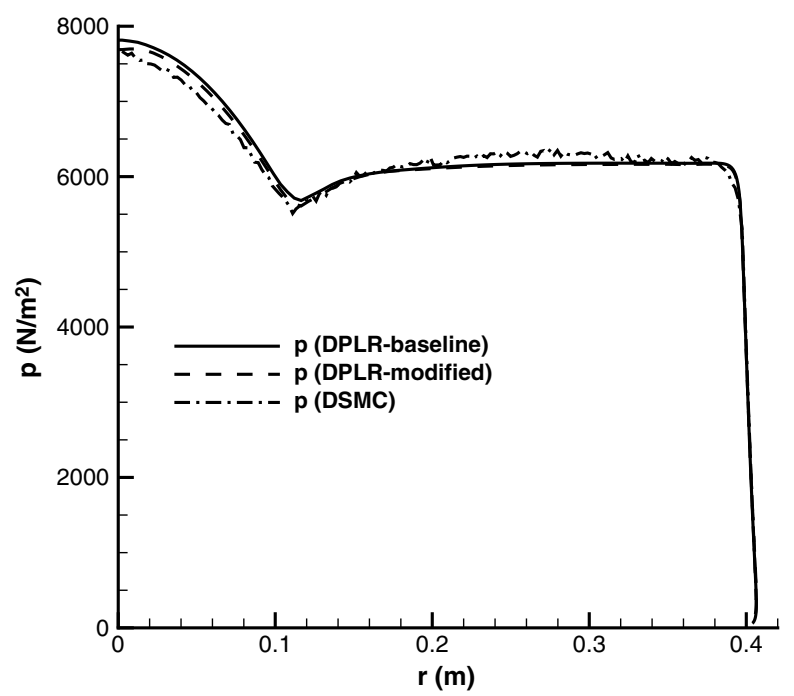

Fig. 22 Profiles of pressure along the surface of the Stardust return capsule at $71 \mathrm{~km}$.

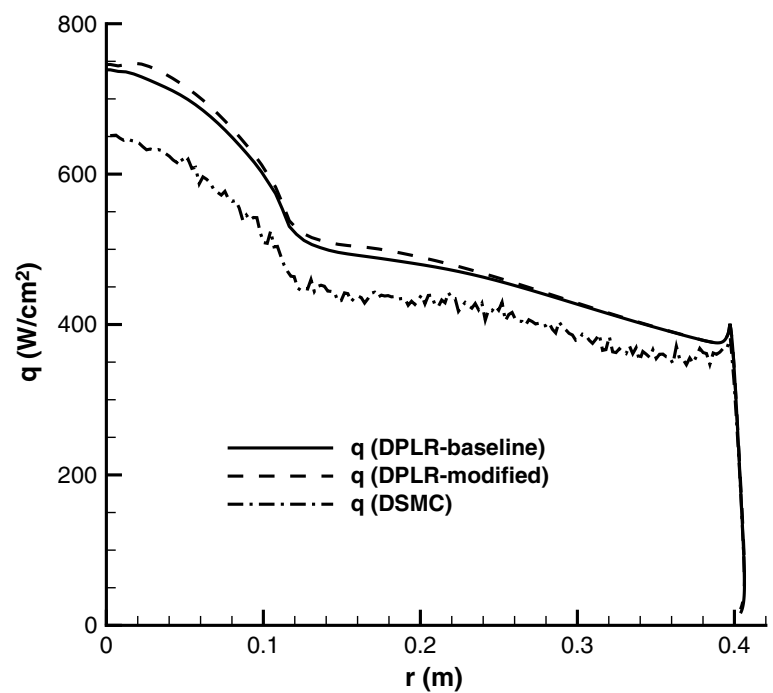

Fig. 23 Profiles of heat flux along the surface of the Stardust return capsule at $71 \mathrm{~km}$.

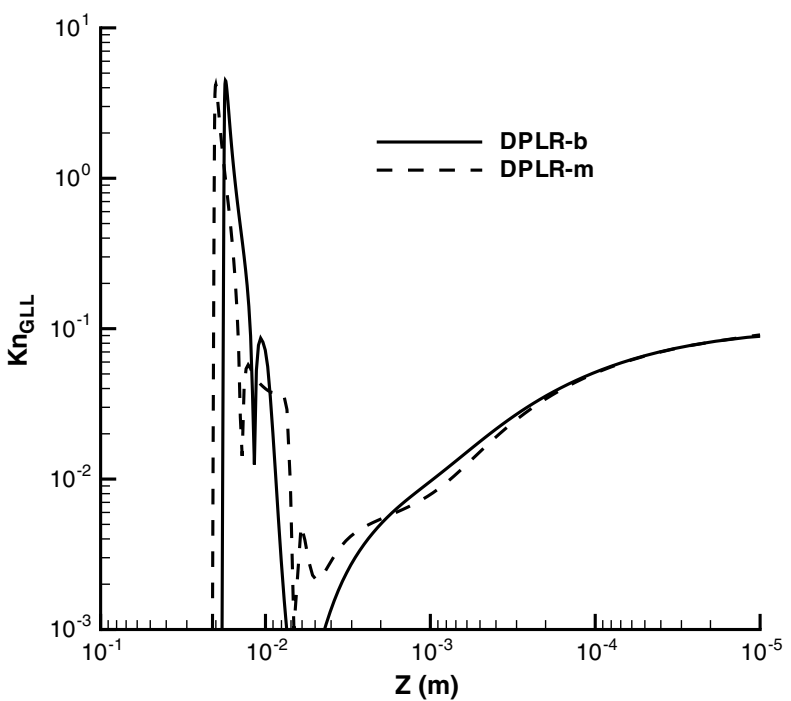

Fig. 24 Profiles of gradient-length local Knudsen number along the stagnation streamline obtained from the two DPLR solutions at $71 \mathrm{~km}$.

lower. Further analysis reveals that the DSMC heat flux contributions from convective heating and surface catalysis are each about $15 \%$ lower than the DPLR contributions. As expected, the agreement between DSMC and DPLR is improved at the lower-altitude condition.

For completeness, the DPLR profiles of gradient-length local Knudsen number based on temperature gradient along the stagnation streamline are shown in Fig. 24. Even though the overall Knudsen number is reduced by a factor of 5 from the $81 \mathrm{~km}$ flow condition, the values of the local Knudsen number at the wall are still large enough to indicate continuum breakdown in this region, although to a lesser extent than at $81 \mathrm{~km}$. For example, using the breakdown criterion of $K n_{\mathrm{GLL}}>0.05$, from the modified DPLR profiles, at $81 \mathrm{~km}$, the region $0.02 \mathrm{~m}>Z>0 \mathrm{~m}$ lies in continuum breakdown. By comparison, at $71 \mathrm{~km}$, the breakdown range is significantly reduced to about $0.0001 \mathrm{~m}>Z>0 \mathrm{~m}$.

\section{Conclusions}

The return trajectory of the Stardust sample return capsule generated the most energetic entry-flight condition of a man-made vehicle that has been flown thus far. The energetic flow condition challenges computational modeling of the flowfield, as the existing thermochemical models for continuum and particle methods have not been calibrated in this highly energetic regime. The present study applied existing CFD (DPLR) and DSMC codes to the Stardust trajectory points at 81 and $71 \mathrm{~km}$, where the overall Knudsen numbers appear to place the flow in the near-continuum regime.

At $81 \mathrm{~km}$, comparisons of baseline DPLR and DSMC solutions showed enormous differences in basic flowfield properties of temperatures and species number densities. For example, the peak translational temperature predicted by DSMC was more than a factor of 2 larger than that predicted by DPLR. The peak plasma density predicted by DPLR was more than a factor of 25 higher than that computed by DSMC. A second DPLR simulation was performed in which the methods employed to determine the controlling temperature in evaluation of the rates for dissociation and ionization were varied. For dissociation, more weight was given to the vibrational temperature, and for ionization, the vibrational temperature was fully used. This approach brought the DPLR chemistry modeling more in line with that employed in DSMC. The results obtained with the modified DPLR chemistry offered significantly improved agreement with the DSMC solutions. This finding illustrates that there are significant uncertainties in the chemical modeling of such energetic flows. It was also noted that the DSMC temperatures always exceeded the DPLR temperatures close to the vehicle surface. 
The surface pressures computed using each of the three simulations were in good agreement. However, there were significant differences in heat flux, with DSMC predicting a peak that was about $40 \%$ lower than that obtained with the modified DPLR simulation. Further analysis revealed that the DSMC convective heat flux was about $25 \%$ lower than the DPLR result, and the DSMC catalytic heat flux was a factor of 2 lower. Evaluation of the local Knudsen number near the stagnation point indicated significant failure of the continuum approach, for which CFD is known to overpredict convective heating. Continuum breakdown at the surface also explains the clear presence in the DSMC solutions of a significant temperature jump at the surface. The elevated DSMC temperatures led to reduced number densities that led to the smaller levels of catalytic heating.

Thus, an important conclusion from this study is that noncontinuum flow effects cannot be accurately estimated from the overall Knudsen number. Careful evaluation of localized continuum breakdown must be made to predict potential limitations of a continuum approach. The very high energy of the Stardust flight produces very high temperatures that in turn lead to very-high-temperature gradients that appear to produce noncontinuum flow at the stagnation point. Another significant conclusion is that the noncontinuum effect of temperature jump can lead to significant reduction in heating from surface chemistry for a fully catalytic material.

As expected, at the higher-density $71 \mathrm{~km}$ condition, the differences between the two DPLR flowfield solutions were almost completely eliminated. There remained some differences from the DSMC results, particularly for temperatures close to the surface and for the ion species number densities. At the surface, the peak heat flux from DSMC was only about $15 \%$ lower than the DPLR results. The local Knudsen number was still found to be sufficiently high to suggest continuum breakdown at the stagnation point, thus again partly explaining the temperature jump and lower heat flux predicted by DSMC.

\section{Acknowledgments}

The work of Iain D. Boyd was sponsored in part by the Stanford Center for Turbulence Research and by the Eloret Corporation through NASA contract NNA04BC25C. Kerry A. Trumble was also supported by NASA contract NNA04BC25C.

\section{References}

[1] Olynick, D., Chen, Y.-K., and Tauber, M. E., "Aerothermodynamics of the Stardust Sample Return Capsule," Journal of Spacecraft and Rockets, Vol. 36, 1999, pp. 442-462. doi:10.2514/2.3466

[2] Mitcheltree, R. A., Wilmoth, R. G., Cheatwood, F. M., Brauckmann, G. J., and Greene, F. A., "Aerodynamics of Stardust Sample Return Capsule," Journal of Spacecraft and Rockets, Vol. 36, 1999, pp. 429 435. doi: $10.2514 / 2.3463$

[3] Wilmoth, R. G., Mitcheltree, R. A., and Moss, J. N., "Low-Density Aerodynamics of the Stardust Sample Return Capsule," Journal of Spacecraft and Rockets, Vol. 36, 1999, pp. 436-441. doi: $10.2514 / 2.3464$

[4] Gupta, R. N., "Aerothermodynamic Analysis of Stardust Sample Return Capsule with Coupled Radiation and Ablation," Journal of Spacecraft and Rockets, Vol. 37, 2000, pp. 507-514. doi: $10.2514 / 2.3592$

[5] Bird, G. A., Molecular Gas Dynamics and the Direct Simulation of Gas Flows, Oxford Univ. Press, Oxford, 1994.

[6] Ozawa, T., Zhong, J., Levin, D. A., Boger, D., and Wright, M. J., "Modeling of the Stardust Reentry Flows With Ionization in DSMC," AIAA Paper 2007-0611, Jan. 2007

[7] Schwartzentruber, T. E., Scalabrin, L. C., and Boyd, I. D., "A Modular Particle-Continuum Method for Hypersonic Non-Equilibrium Gas Flows," Journal of Computational Physics, Vol. 225, 2007, pp. 1159_ 1174 doi:10.1016/j.jcp.2007.01.022

[8] Boyd, I. D., and Jenniskens, P. J., "Modeling of Stardust Entry at High Altitude, Part 2: Radiation Analysis," Journal of Spacecraft and Rockets (to be published).

[9] Lofthouse, A. J., Scalabrin, L. C., and Boyd, I. D., "Velocity Slip and
Temperature Jump in Hypersonic Aerothermodynamics," Journal of Thermophysics and Heat Transfer, Vol. 22, No. 1, 2008, pp. 38-49. doi: $10.2514 / 1.31280$

[10] Wright, M., Loomis, M., and Papadopoulos, P., "Aerothermal Analysis of the Project Fire II Afterbody Flow," Journal of Thermophysics and Heat Transfer, Vol. 17, No. 2, 2003, pp. 240-249. doi:10.2514/2.6757

[11] Wright, M., Candler, G., and Bose, D., "Data-Parallel Line Relaxation Method for the Navier-Stokes Equations," AIAA Journal, Vol. 36, No. 9, 1998, pp. 1603-1609. doi:10.2514/2.586

[12] MacCormack, R., and Candler, G., "The Solution of the Navier-Stokes Equations Using Gauss-Seidel Line Relaxation," Computers and Fluids, Vol. 17, No. 1, 1989, pp. 135-150. doi:10.1016/0045-7930(89)90012-1

[13] Yee, H., "A Class of High-Resolution Explicit and Implicit Shock Capturing Methods," NASA TM 101088, Feb. 1989.

[14] Wercinski, P., Chen, Y.-K., Loomis, M., Tauber, M., McDaniel, R., Wright, M., Papadopoulos, P., Allen, G., and Yang, L., "Neptune Aerocapture Entry Vehicle Preliminary Design," AIAA Paper 20024812, Aug. 2002.

[15] Wright, M., Bose, D., and Olejniczak, J., "The Impact of Flowfield Radiation Coupling on Aeroheating for Titan Aerocapture," Journal of Thermophysics and Heat Transfer, Vol. 19, No. 1, 2005, pp. 17-27. doi:10.2514/1.10304

[16] Gupta, R., Yos, J., Thompson, R., and Lee, K., "A Review of Reaction Rates and Thermodynamic and Transport Properties for an 11-Species Air Model for Chemical and Thermal Nonequilibrium Calculations to 30000 K," NASA RP-1232, Aug. 1990.

[17] Palmer, G. E., and Wright, M. J., "A Comparison of Methods to Compute High Temperature Gas Viscosity," Journal of Thermophysics and Heat Transfer, Vol. 17, No. 2, 2003, pp. 232-239. doi:10.2514/2.6756

[18] Palmer, G. E., and Wright, M. J., "A Comparison of Methods to Compute High Temperature Gas Thermal Conductivity," AIAA Paper 2003-3913, Jun. 2003.

[19] Bartlett, E. P., Kendal, R. M., and Rindal, R. A., "An Analysis of the Coupled Chemically Reacting Boundary Layer and Charring Ablator: Part 4-A Unified Approximation for Mixture Transport Properties for Multicomponent Boundary-Layer Applications," NASA CR-1063, June 1968.

[20] Parker, J. G., "Rotational and Vibrational Relaxation in Diatomic Gases," Physics of Fluids, Vol. 2, 1959, pp. 449-462. doi:10.1063/1.1724417

[21] Millikan, R., and White, D., "Systematics of Vibrational Relaxation," Journal of Chemical Physics, Vol. 39, No. 12, 1963, pp. 3209-3213. doi:10.1063/1.1734182

[22] Park, C., Nonequilibrium Hypersonic Aerothermodynamics, Wiley, New York, 1990.

[23] Park, C., "Review of Chemical-Kinetic Problems of Future NASA Missions, 1: Earth Entries," Journal of Thermophysics and Heat Transfer, Vol. 7, No. 3, 1993, pp. 385-398. doi: $10.2514 / 3.431$

[24] Bose, D., and Candler, G., "Thermal Rate Constants of the $\mathrm{N}_{2}+\mathrm{O}=$ $\mathrm{ED} \mathrm{NO}+\mathrm{N}$ Reaction Using Ab Initio 3A" and 3A' Potential Energy Surfaces," Journal of Chemical Physics, Vol. 104, No. 8, 1996, pp. $2825-2833$. doi:10.1063/1.471106

[25] Boyd, I. D., and Gokcen, T., "Computation of Axisymmetric and Ionized Hypersonic Flows Using Particle and Continuum Method," AIAA Journal, Vol. 32, 1994, pp. 1828-1837. doi: $10.2514 / 3.12180$

[26] Boyd, I. D., "Analysis of Rotational Nonequilibrium in Standing Shock Waves of Nitrogen," AIAA Journal, Vol. 28, 1990, pp. 1997-1999. doi: $10.2514 / 3.10511$

[27] Boyd, I. D., "Analysis of Vibrational-Translational Energy Transfer Using the Direct Simulation Monte Carlo Method," Physics of Fluids A, Vol. 3, 1991, pp. 1785-1791. doi: $10.1063 / 1.857959$

[28] Haas, B. L., and Boyd, I. D., "Models for Direct Monte Carlo Simulation of Coupled Vibration-Dissociation," Physics of Fluids A, Vol. 5, 1993, pp. 478-489. doi: $10.1063 / 1.858870$

[29] Bondar, Y., and Ivanov, M., "DSMC Dissociation Model Based on Two-Temperature Chemical Rate Constant," AIAA Paper 2007-0614, Jan. 2007.

[30] Wilson, J., "Ionization Rate of Air Behind High-Speed Shock Waves," Physics of Fluids, Vol. 9, 1966, pp. 1913-1921. doi:10.1063/1.1761543 
[31] Boyd, I. D., "Modeling Backward Chemical Rate Processes in the Direct Simulation Monte Carlo Method," Physics of Fluids, Vol. 19, 2007, Paper 126103. doi: $10.1063 / 1.2825038$

[32] Bird, G. A., "Nonequilibrium Radiation During Re-Entry at 10 km/s," AIAA Paper 87-1543, June 1987.

[33] Boyd, I. D., "Monte Carlo Simulation of Nonequilibrium Flow in Low Power Hydrogen Arcjets," Physics of Fluids, Vol. 9, 1997, pp. 30863095. doi:10.1063/1.869474

[34] Wang, W.-L., and Boyd, I. D., "Predicting Continuum Breakdown in Hypersonic Viscous Flows," Physics of Fluids, Vol. 15, 2003, pp. 91100 .

doi: $\underline{10.1063 / 1.1524183}$

D. Kontinos Guest Editor 